\title{
Du paradoxe au style paradoxal : l'exemple des Caractères de La Bruyère
}

From Paradox to Paradoxal Style: La Bruyère's Les Caractères

\section{Pierre-Yves Gallard}

\section{(2) OpenEdition}

1 Journals

\section{Édition électronique}

URL : http://journals.openedition.org/pratiques/2548

DOI : $10.4000 /$ pratiques. 2548

ISSN : 2425-2042

Éditeur

Centre de recherche sur les médiations (CREM)

\section{Référence électronique}

Pierre-Yves Gallard, « Du paradoxe au style paradoxal : l'exemple des Caractères de La Bruyère », Pratiques [En ligne], 165-166 | 2015, mis en ligne le 01 octobre 2015, consulté le 10 décembre 2020. URL : http://journals.openedition.org/pratiques/2548; DOI : https://doi.org/10.4000/pratiques.2548

Ce document a été généré automatiquement le 10 décembre 2020.

(c) Tous droits réservés 


\title{
Du paradoxe au style paradoxal : l'exemple des Caractères de La Bruyère
}

From Paradox to Paradoxal Style: La Bruyère's Les Caractères

\author{
Pierre-Yves Gallard
}

\section{Introduction}

1 Pour interroger les interactions entre figure et contexte, nous proposons de travailler sur l'écriture du paradoxe dans Les Caractères de La Bruyère. Ce corpus, marqué par un souci d'appropriation de l'éloquence classique aux codes de la mondanité, se caractérise par un rapport ambivalent à la rhétorique. Esthétique de la pointe et gout du naturel soumettent en effet le discours moraliste à des impératifs souvent contradictoires et imposent à l'auteur un exercice d'équilibre précaire : celui de veiller à l'expressivité de son écriture sans tomber dans l'extravagance affectée, de cultiver un art du bien dire qui ne verse ni dans la grandiloquence pédante, ni dans l'obscurité du «bel esprit ». L'idéal prôné est celui d'un échange délié, conciliant et sans aspérité ; le discours peut être subtil, mais il ne doit en aucun cas sombrer dans l'hermétisme.

Dans ce contexte, produire un paradoxe, c'est contrevenir aux règles de transparence et de fluidité censées régir la communication et, par là même, s'exposer au ridicule. En outre, la longue tradition scolastique qui est attachée au paradoxe contribue à accabler cette forme aux yeux du public : le tour serait artificiel et sophistique, il appartiendrait à l'arsenal grandiloquent d'une rhétorique désuète. Le paradoxe, qui repose sur l'actualisation discursive d'une contradiction, s'impose pourtant comme un outil privilégié pour dévoiler l'inconséquence des comportements humains. Et de fait, à la lecture, le paradoxe apparait comme un motif fondamental de l'œuvre.

3 Nous étudierons les stratégies développées par J. de La Bruyère pour intégrer le paradoxe à l'économie galante de la conversation. Dissimulation de sa binarité formelle, 
implicitation de la contradiction qu'il renferme, hybridation de la figure... : le moraliste infléchit le "schème discursif » paradoxal (Bonhomme, 2005 : 38) afin de l'approprier aux codes esthétiques de l'honnêteté mondaine. Nous verrons que ces infléchissements imposés à la forme prototypique du paradoxe, loin de conduire à la dissolution de la pensée paradoxale dans le discours moraliste, permettent au contraire l'émergence de ce que nous proposons d'appeler un «style paradoxal». Il s'agit de mettre en évidence par ce titre que les contraintes imposées par le contexte culturel à la production des énoncés paradoxaux, plutôt que d'entrainer la disparition de la figure, confèrent à son écriture une forme plus souple et une efficacité critique nouvelle. Nous tâcherons de montrer que la diversification des formes du paradoxe sous la pression du contexte conduit à faire de cette figure une forme matricielle, qui soutient le développement d'une certaine attitude intellectuelle, oriente une pratique d'écriture et unifie un style à l'échelle du recueil.

\section{Paradoxe et conversation}

$\mathrm{K}$. Wołowska (2008) définit le paradoxe de langue comme un phénomène dialectique qui consiste en l'actualisation discursive d'une contradiction et en son assimilation par le discours. La contradiction repose sur la jonction syntagmatique de sèmes opposés ; son assimilation par le discours implique des procédés interprétatifs d'assimilation et de dissimilation sémantique. Prenons un exemple :

"Les hommes souvent veulent aimer, et ne sauraient y réussir; ils cherchent leur défaite sans pouvoir la rencontrer; et si j'ose ainsi parler, ils sont contraints de demeurer libres » (Chapitre IV, § 16, nous soulignons).

La contradiction paradoxale repose sur la jonction des sèmes /contrainte/ et /liberté/ inhérents à contraints et à libres. La neutralisation de la contradiction nécessite quant à elle l'interprétation métaphorique du terme libres. Dans sa forme prototypique, le paradoxe se caractérise donc par une structure binaire d'opposition-jonction qui lui confère une saillance forte.

6 Cette configuration marquée peut aisément passer pour de la pompe rhétorique et contrarier le goût du naturel, car elle tend par son fonctionnement même à outrer les contrastes. Le paradoxe souffre par ailleurs d'une mauvaise réputation: associé dans la mémoire collective aux exercices des pyrrhoniens, aux syllogismes des sophistes et à l'enseignement de la dialectique par la scolastique médiévale, il est régulièrement condamné pour son caractère jugé artificiel et pédant ${ }^{1}$. Or, l'ethos recherché par La Bruyère n'est ni celui du maitre de rhétorique, ni celui de l'esprit fort; le moraliste se présente au contraire comme un homme du monde, qui observe et qui remarque : il s'agit de « mesure[r] au compas les grandeurs et les proportions » (chap. III, § 48). La remarque que nous avons citée précédemment fournit d'ailleurs une belle illustration des précautions que prend La Bruyère lorsqu'il recourt à une formule ostensiblement paradoxale. Le paradoxe final - «ils sont contraints de demeurer libres»- est un exemple rare dans le recueil: celui d'une contradiction explicite, assumée par l'énonciateur et qui relève du jeu de mots. Aussi le moraliste semble-t-il presque s'excuser de céder à cette facilité et commente, en incise: "Si j'ose ainsi parler». La modalité autonymique a pour vocation de suturer la «faille» ouverte par la contradiction ${ }^{2}$ et d'aiguiller la réception de la figure, en soulignant qu'elle relève d'une manière de parler, et non d'un vice de raisonnement. En outre, ce paradoxe est préparé et comme introduit par un premier énoncé, lui aussi paradoxal, mais plus discret: "Ils cherchent leur 
défaite ». Le moraliste procède donc par gradation, afin de rendre le paradoxe final plus acceptable dans l'esprit du lecteur. Notons enfin que La Bruyère s'autorise ici d'un motif paradoxal répandu et couramment admis: celui de la prison délicieuse, du doux asservissement qu'est l'amour.

\section{Des stratégies d'intégration de la figure au discours moraliste}

7 Le moraliste travaille ainsi à adoucir l'âpreté du paradoxe, à en atténuer l'artificialité afin de le rendre acceptable pour ses lecteurs. Parmi les différentes stratégies mises en place pour intégrer le paradoxe au discours moraliste, certains procédés reviennent régulièrement.

\subsection{Désamorcer la contradiction}

8 L'un d'entre eux consiste à désamorcer la contradiction, par l'insertion de gloses détensives ou par le biais de ce que C. Costentin (2003: 182) a baptisé des «lexèmes de compensation ", dont le rôle est d'atténuer l'effet de contradiction. Prenons pour exemple cette remarque :

«Cesser d'aimer, preuve sensible que l'homme est borné, et que le cœur a ses limites.

C'est faiblesse que d'aimer : c'est souvent une autre faiblesse que de guérir.

On guérit comme on se console : on n'a pas dans le cœur de quoi toujours pleurer et toujours aimer » (chapitre IV, § 34).

$\mathrm{Au}$ cœur de la remarque, un paradoxe : la même cause (la faiblesse) entraine deux effets opposés (aimer/cesser d'aimer). Mais la contradiction est doublement nuancée et désamorcée: d'abord de manière interne, par le biais de l'adverbe souvent et du groupe déterminatif une autre, qui agissent comme "lexèmes de compensation " et rompent l'opposition symétrique des deux propositions antithétiques; puis de manière externe, par l'insertion du paradoxe entre deux gloses explicatives, qui offrent une résolution à la contradiction (l'homme est borné aussi bien dans sa capacité de résistance que dans sa faculté d'abandon à l'amour). Il semblerait que le moraliste recherche le point d'équilibre, entre refus d'une généralisation excessive et volonté de maintenir la tension paradoxale qui confère à la remarque toute son expressivité.

\subsection{Dissimuler la binarité de la figure}

10 Pour préserver cet équilibre, La Bruyère intercale souvent entre les deux termes de la contradiction une troisième option, dont l'effet est de dissimuler la structure binaire du paradoxe: la figure devient plus difficile à isoler, elle est donc moins susceptible de passer pour un artifice rhétorique et s'intègre plus aisément au propos.

"Que faire d'Egesippe qui demande un emploi ? [...] il est propre à tout, disent ses amis, ce qui signifie toujours qu'il n'a pas plus de talent pour une chose que pour une autre, ou en d'autres termes, qu'il n'est propre à rien » (chapitre II, §10, nous soulignons).

11 Le paradoxe nait de l'équivalence que pose le moraliste entre deux énoncés violemment contradictoires. Toutefois, l'insertion d'une troisième option entre «tout » et « rien » 
assure la transition et relègue le caractère spectaculaire ou péremptoire de la figure au second plan : ce qui est mis en valeur ici, c'est le travail de décryptage du discours tenu par les « amis ", la subtilité d'une ironie discrète, plutôt que l'éclat d'un tour sentencieux. Notons enfin que le paradoxe s'intègre à ce que M. Bonhomme (2005:56) appelle « un schème figural englobant", qui relève à la fois de l'ironie, de la paradiastole et du paradoxe. La figure, moins immédiatement identifiable, est donc moins susceptible de passer pour une formule.

\subsection{Recourir à l'implicite}

12 Une troisième stratégie mise en œuvre par La Bruyère pour intégrer le paradoxe au discours moraliste réside dans l'implicitation de la contradiction paradoxale. La remarque suivante nous offre un bel exemple de ce procédé :

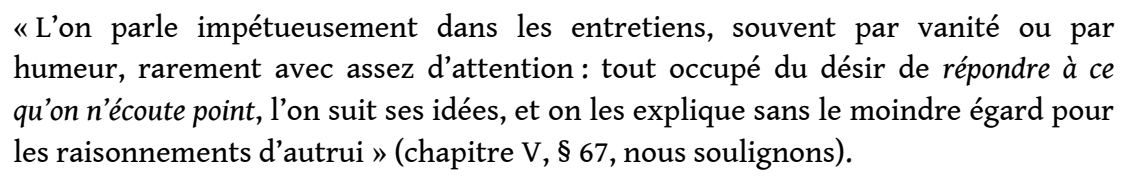

L'exemple est d'autant plus intéressant que les éditions 1 à 4 des Caractères donnaient une variante: "tout occupé du désir de répondre à ce que l'on ne se donne même pas la peine d'écouter ». La correction de la cinquième édition fait apparaitre la logique d'implicitation qui nous semble orienter l'écriture du paradoxe dans Les Caractères : au-delà d'un simple souci d'économie de moyens ou d'élégance du propos, cette réécriture révèle un effort de La Bruyère pour supprimer ce qui souligne trop explicitement l'aberration logique (dans notre exemple, la locution verbale se donner la peine de, renforcée par l'adverbe même). Le paradoxe est donc moins immédiatement repérable comme forme, mais son effet n'est pas moindre: le soin d'actualiser la contradiction entre répondre et ne pas écouter est davantage laissé au lecteur. Ce dernier, flatté d'avoir remarqué, puis résolu une difficulté, est plus enclin à adhérer au propos tenu. Aussi cette remarque témoigne-t-elle de la parfaite intégration du paradoxe à l'esthétique de la conversation, dont l'esprit, nous dit La Bruyère, "consiste bien moins à en montrer beaucoup qu'à en faire trouver aux autres » (chap. V, § 16).

\section{Appropriation de la figure aux codes de l'esprit mondain et diversification des formes du paradoxe}

14 Jouant de la plasticité qui caractérise le schème discursif paradoxal, le moraliste approprie la figure aux codes de l'esprit mondain. Le paradoxe se fond ainsi dans diverses formes de productions galantes, telles l'énigme ${ }^{3}$ ou les jeux de distinguo précieux :

«Qu'est-ce qu'une femme que l'on dirige? est-ce une femme plus complaisante pour son mari, plus douce pour ses domestiques, plus appliquée à sa famille et à ses affaires, plus ardente et plus sincère pour ses amis ; qui soit moins esclave de son humeur, moins attachée aux commodités de la vie; je ne dis pas qui fasse des largesses à ses enfants qui sont déjà riches, mais qui opulente elle-même et accablée $\mathrm{du}$ superflu leur fournisse le nécessaire, et leur rende au moins la justice qu'elle leur doit; qui soit plus exempte d'amour de soi-même et d'éloignement pour les autres, qui soit plus libre de tous attachements humains? non, dites-vous, ce n'est rien de toutes ces choses; j'insiste, et je vous demande qu'est-ce donc qu'une 
femme que l'on dirige? je vous entends, c'est une femme qui a un directeur » (chap. III, § 36). engage l'interprétation paradoxale du mot directeur: les directeurs ne dirigent pas la conduite de celles qu'ils sont censés conseiller. Le paradoxe est moins explicite que dans les exemples précédents, dans la mesure où le lecteur peine à isoler une contradiction franche entre deux termes de l'énoncé : la structure d'opposition-jonction qui caractérise le paradoxe semble ici dilatée, étirée du début à la fin de la remarque. Son actualisation n'en est pas moins nécessaire à la compréhension de cette remarque et de celles qui suivent, consacrées elles aussi à la direction de conscience. Le moraliste engage ainsi le lecteur à repérer les contradictions que masque l'illusoire transparence du langage et, partant, à penser hors des cadres préconstruits et des idées reçues. C'est pourquoi il importe de ne pas réduire le travail d'intégration du paradoxe à une quelconque « domestication » de l'esprit paradoxal, ni à son effacement devant les règles impérieuses de « l'esprit de la conversation » $(\mathrm{V}, 16)$. Le travail de la forme auquel se livre le moraliste ne nous parait nullement entrainer la dissolution de la pensée paradoxale, mais bien sa diffusion. L'exemple qui suit montre d'ailleurs comment la pratique moraliste du paradoxe infléchit le discours galant : appelé jalousie ; cette autre jalousie qui est un sentiment juste, naturel, fondé en raison et sur l'expérience, mériterait un autre nom. lon fait souffrir les autres.

«Celles qui ne nous ménagent sur rien, et ne nous épargnent nulles occasions de jalousie, ne mériteraient de nous aucune jalousie, si l'on se réglait plus par leurs sentiments et leur conduite que par son cœur » (chap. IV, § 29, nous soulignons).

19 cette pratique répond à un désir d'ordonner le multiple, mais il semblerait que le moraliste se plaise ici à raffiner la complexité, sans stabiliser le classement qu'il entreprend. La remarque s'ouvre sur le constat d'une ambigüité : le mot jalousie semble désigner deux sentiments opposés. Le moraliste distingue alors la délicatesse, qui serait le signe d'un attachement vif et sincère, de la jalousie, qui constituerait une perversion odieuse de la délicatesse. Toutefois, le dernier paragraphe amalgame à nouveau jalousie et délicatesse en cette formule: "Celles qui [...] ne nous épargnent nulle occasion de jalousie ne mériteraient de nous aucune jalousie ». Le paradoxe repose sur la jonction du sème évaluatif /positif/ afférent au sémème "mériter » et du sème évaluatif /négatif/ inhérent au sémème «jalousie ». Mais cette contradiction est elle-même redoublée par un paradoxe que présuppose l'emploi du conditionnel: si le moraliste peut écrire "[ces femmes] ne mériteraient de nous aucune jalousie ", c'est qu'elles en sont gratifiées. La Bruyère pointe ici la nature paradoxale du sentiment amoureux : nous faisons dépendre notre bonheur des êtres qui nous tourmentent. Aussi l'effort déployé dans cette remarque pour chasser l'équivoque échoue-t-il: le distinguo entrepris dans les premiers paragraphes s'abime dans la complexité du sentiment amoureux.

20

Cette dynamique peut être commentée de deux façons. La première consiste à rappeler que l'esthétique précieuse du distinguo réprouve toute forme de pédantisme : s'il importe 
de faire des observations justes et profondes, il faut se garder d'être excessivement analytique ou pédagogue. Dans cette perspective, le paradoxe du dernier paragraphe aurait pour vocation de soutenir la légèreté du propos, le ton galant de la conversation. Une autre interprétation possible décèle dans cette remarque un déport, voire une subversion de la tradition précieuse du distinguo. La Bruyère ne chercherait pas à stabiliser son discours; au contraire, il inviterait son lecteur à faire l'expérience du caractère intrinsèquement contradictoire $d u$ réel: la tentative d'ordonnancement demeure alors inachevée, la critique du discours laisse place au constat d'une contradiction indépassable.

\section{Une forme matricielle : l'élaboration d'un « style paradoxal »}

21 L'exemple que nous venons d'analyser montre que si l'appropriation de la figure à l'esthétique conversationnelle entraine une diversification des formes du paradoxe, elle ne peut être assimilée à un quelconque affaiblissement de l'esprit paradoxal. L'intégration de la figure au discours moraliste n'entame ni la cohérence de la configuration globale qui caractérise la figure, ni son dynamisme fonctionnel. Le schème paradoxal ne perd donc rien de sa capacité à organiser et à orienter un discours; il nous apparait au contraire comme une forme matricielle, capable de structurer un regard, d'orienter un discours et d'unifier un style.

\subsection{Structuration d'un regard « para-doxal »}

Le projet de La Bruyère nous semble en effet procéder d'une vision " para-doxale », c'està-dire attentive aux incohérences et aux contradictions qui dénoncent la finitude de l'homme. Comme le souligne E. Bury (1995: 28-29) dans la préface qu'il consacre à son édition des Caractères, faire apparaitre ces contradictions est une question d'optique : « La distance et la perspective jouent un grand rôle dans la perception du réel, et la métaphore optique [est] constante dans les Caractères.» Le paradoxe nous apparait précisément comme un moyen privilégié de transcrire et d'informer ces jeux de perspective et de focalisation. L'exemple qui suit permet d'observer les processus de sélection et de cadrage qui déterminent le point de vue paradoxal du moraliste :

"L'on est petit à la Cour, et quelque vanité que l'on ait, on s'y trouve tel; mais le

mal est commun, et les grands mêmes y sont petits » (chap. VIII, § 5).

Cette remarque s'ouvre sur une proposition fort courte: «l'on est petit à la Cour ». Le pronom personnel indéfini on ainsi que le présent de vérité générale confèrent au propos un haut degré d'abstraction et de généralité. Le lecteur dispose donc de peu d'indices pour élucider cette assertion surprenante, qui associe de manière inattendue la Cour à la petitesse. La suite de la remarque reformule l'affirmation surprenante et en explicite le caractère contradictoire. Ainsi, par un effet de sélection et de rapprochement, le paradoxe final concentre la tension sur deux termes coréférents: grands et petits. L'énoncé substitue par ailleurs au verbe attributif se trouver, la copule être, ce qui crée un effet d'objectivité. Le paradoxe, qui nait de la jonction des termes contradictoires au moyen de la copule, apparait donc comme le résultat d'un travail de "cadrage »: la contradiction est réduite - par abstraction du contexte et généralisation du propos - à son expression la plus simple et la plus radicale. Pour la résorber, le lecteur doit rétablir 
le contexte manquant. Il procède alors à une série d'opérations interprétatives qui font de la petitesse une qualité relative à un lieu spécifique - la Cour - et à un référent d'une grandeur inégalable : le roi. En retour, la grandeur supposée des « Grands » est elle-même mise en perspective. Le paradoxe soutient donc une réorganisation de l'échelle sociale des valeurs, adossée à un processus de défigement de la langue.

Le paradoxe s'impose ainsi comme un motif fondamental des Caractères. En tant que mise en œuvre discursive d'une contradiction logique, il constitue une forme-sens, capable de prolonger dans l'écriture le regard critique du moraliste et de répondre à l'ambition "para-doxale » de La Bruyère : celle de saisir les ridicules et les contradictions pour les fixer dans l'espace d'une formule, ou les dépeindre à l'échelle d'une remarque. Le changement de paradigme que nous proposons en titre, «du paradoxe au style paradoxal ", permet de rendre compte de cette dynamique : la figure s'émancipe du cadre de la phrase, où le confinent souvent les dictionnaires de rhétorique, pour se déployer à l'échelle du paragraphe, voire de la page. Il se fait alors principe d'écriture, et préside à la composition de bon nombre de remarques - quand bien même celles-ci ne présentent pas de contradictions apparentes.

\subsection{Du syntagme à la page}

Nous illustrerons ce parcours allant du syntagme à la page par l'étude de deux remarques, représentatives des actualisations «microstructurale» et "macrostructurale» de la figure ${ }^{4}$. La première témoigne d'un travail de reprise et de variation autour d'un même paradoxe, reproduit sous des formes plus ou moins condensées :

«Celui-là est riche, qui reçoit plus qu'il ne consume; celui-là est pauvre dont la dépense excède la recette.

Tel avec deux millions de rente peut être pauvre chaque année de cinq cent mille livres.

Il n'y a rien qui se soutienne plus longtemps qu'une médiocre fortune ; il n'y a rien dont on voie mieux la fin que d'une grande fortune.

L'occasion prochaine de la pauvreté, c'est de grandes richesses.

S'il est vrai que l'on soit riche de tout ce dont on n'a pas besoin, un homme fort riche, c'est un homme qui est sage.

S'il est vrai que l'on soit pauvre par toutes les choses que l'on désire ; l'ambitieux et

l'avare languissent dans une extrême pauvreté » (chap. VI, § 49).

La remarque est composée comme suit : à l'énoncé d'une loi générale sur la richesse et la pauvreté succèdent trois versions du paradoxe que cette loi fait surgir ; deux paragraphes viennent alors tirer les conclusions de ces observations, en déplaçant la question de la richesse et de la pauvreté du plan matériel au plan moral et philosophique. L'énoncé de la loi générale est marqué par un souci de rigueur et de clarté, que soulignent la reprise de la construction syntaxique "celui-là est ... [+ relative] » et le chiasme sémantique (formé par les mots reçoit, consume, dépense et recette). Ce système d'anaphores et de symétries rythme l'ensemble de la remarque; il permet l'élaboration d'un cadre rhétorique dont l'équilibre et la régularité structurent le développement et l'amplification du paradoxe.

La première formulation de ce dernier ( Tel avec deux millions de rente peut être pauvre chaque année de cinq cent mille livres») témoigne d'une certaine prudence: non seulement la relation attributive est modalisée par le semi-auxiliaire pouvoir, mais le complément de l'adjectif pauvre vient apporter une précision qui rend l'énoncé plus acceptable, en faisant de la "pauvreté » ainsi décrite une pauvreté relative et non 
absolue. La seconde formulation pousse le paradoxe un peu plus loin, en défendant la supériorité des fortunes médiocres sur les grandes fortunes: "Il n'y a rien qui se soutienne plus longtemps qu'une médiocre fortune ; il n'y a rien dont on voie mieux la fin que d'une grande fortune ». Certes, cette supériorité n'est envisagée que du point de vue de la longévité ; certes, le lecteur des Caractères est habitué à la revalorisation du médiocre aux dépens de l'excellent: la position n'en est pas moins difficile à défendre. Aussi le moraliste recourt-il à la même construction que précédemment, pour faire valoir la cohérence inattaquable de son raisonnement : l'anaphore « il n'y a rien qui... », « il n'y a rien dont... » reprend jusqu'à l'ordre des pronoms relatifs de la première phrase. Ces deux premières variations permettent à La Bruyère de préparer l'esprit de son lecteur à la phrase suivante, qui représente l'actualisation discursive la plus contradictoire du paradoxe développé : «L'occasion prochaine de la pauvreté, c'est de grandes richesses ». L'énoncé se caractérise par son abstraction : il ne s'agit plus d'associer «trente millions de rente » au fait d'être endetté, ni même " une grande fortune » à une fortune épuisée, mais « la pauvreté » à « de grandes richesses ». Les expressions circonstanciées laissent place à une simple paire d'antonymes, dont l'opposition semble indépassable. C'est alors que se déploie toute l'ironie de l'auteur à l'encontre du terme d'« occasion prochaine » : le concept emprunté au vocabulaire théologique des jésuites, apparait ici comme le vecteur des paradoxes les plus invraisemblables, capable de poser un rapport d'équivalence entre les notions les plus contraires 5 .

$\mathrm{Au}$ travail de resserrement et de concentration dont témoigne cette remarque répond un travail symétrique d'expansion et de dilatation de la structure qui caractérise le paradoxe. La remarque qui suit offre l'exemple d'un déploiement de la figure non à l'intérieur d'un syntagme (comme c'est le cas pour les oxymores) ni même d'une phrase (comme nous venons de l'envisager), mais à l'échelle d'une remarque entière :

«Une tenue d'états, ou les chambres assemblées pour une affaire très capitale, n'offrent point aux yeux rien de si grave et de si sérieux, qu'une table de gens qui jouent un grand jeu; une triste sévérité règne sur leurs visages; implacables l'un pour l'autre et irréconciliables ennemis pendant que la séance dure, ils ne reconnaissent plus ni liaisons, ni alliance, ni naissance, ni distinctions : le hasard seul, aveugle et farouche divinité, préside au cercle et y décide souverainement; ils l'honorent tous par un silence profond, et par une attention dont ils sont partout ailleurs fort incapables : toutes les passions comme suspendues cèdent à une seule ; le courtisan alors n'est ni doux, ni flatteur, ni complaisant, ni même dévot" (chap. VI, § 72).

Le paradoxe opère à l'échelle du paragraphe. Il consiste à décrire le jeu comme une activité grave et sérieuse au point de paraitre plus âpre que la guerre. Le moraliste joue avec les attentes du lecteur: tandis que l'évocation des affaires politiques du Royaume donne au propos un air de solennité, les adjectifs modalisés en intensité (« une affaire très capitale ", " rien de si grave et de si sérieux ») et le rythme croissant de la phrase (6,15 et 15 syllabes) semblent annoncer l'évocation d'un sujet élevé. Aussi le contraste est-il grand, quand la comparaison s'achève de manière déceptive sur l'évocation d'une table de jeu. Les propositions suivantes, qui décrivent la «triste sévérité » des joueurs, exacerbent encore la tension entre la réalité décrite et les termes employés. Les métaphores guerrières suggèrent en effet que l'ardeur du combat est plus forte autour de la table de jeu que sur le champ de bataille - où, malgré la violence des affrontements, les « liaisons ", les « alliances » et les rangs sociaux sont théoriquement respectés. Objet de la passion des joueurs, le jeu désagrège donc les liens sociaux qu'il est censé entretenir; le cercle, dont la conduite n'est plus réglée par la recherche commune du plaisir, est 
abandonné à la conduite de cette "farouche divinité » qu'est le hasard - de manière symptomatique, la conversation laisse place alors à « un silence profond ». Le moraliste achève plaisamment son tableau par l'évocation d'une chimère monstrueuse : le courtisan « ni doux, ni flatteur, ni complaisant, ni même dévot », c'est-à-dire dépourvu de toutes les propriétés qui le définissent habituellement. L'auteur dépeint un renversement si complet des attitudes sociales et des valeurs qui sont traditionnellement attachées au jeu qu'il se voit contraint de recourir aux caractérisations les plus contraires à l'usage.

Ainsi, bien que ce paragraphe ne renferme pas de contradiction en bonne et due forme, il nous semble que la logique qui préside à sa construction est bien celle du paradoxe. Contrairement aux occurrences que nous avons relevées au début de ce travail, le paradoxe ne résulte plus de l'exhibition d'une contradiction franche entre deux termes conjoints (comme c'était le cas pour «ils sont contraints de demeurer libres»), mais procède d'un «travail de la discordance »: l'ensemble de la remarque vise à creuser l'écart entre des champs notionnels incompatibles et néanmoins associés, à faire apparaitre une disproportion inexplicable, à souligner la disconvenance entre l'attitude des joueurs d'une part et l'activité à laquelle ils se livrent d'autre part. L'effet de paradoxe se déplace, mais n'est pas moins fort que dans le cas des oppositions binaires : la tension sémantique gagne en amplitude ce qu'elle perd en intensité. En effet, si la jonction de deux antonymes concentre la contradiction de manière spectaculaire sur une petite portion du discours, la dilatation du paradoxe à l'échelle d'un paragraphe impose au récepteur d'attendre plus longtemps avant de voir la tension s'apaiser et le contraint à un suspens interprétatif plus long.

31 Cet exemple nous parait illustrer la dernière étape du travail d'acclimatation conversationnelle du paradoxe: le schème paradoxal informe la remarque dans son ensemble, sans se cristalliser en une contradiction explicite qui, par son caractère saillant, détachable - « décontextualisable ${ }^{6}$ » - viendrait rompre la fluidité de l'écriture et perturber le bon déroulement de la communication.

\section{Conclusion}

Concluons donc sur ce constat: les Caractères témoignent de la parfaite intégration du paradoxe à l'esthétique de la conversation. Attaché à saisir les ridicules et à révéler les contradictions, et en même temps soucieux de respecter les codes de l'honnêteté mondaine, La Bruyère approprie la figure à un contexte culturel qui condamne fermement toute forme de grandiloquence. Cette « acclimatation conversationnelle » du paradoxe passe par un travail de la forme: la contradiction paradoxale est tantôt dissimulée, tantôt modulée, modalisée, implicitée... Derrière la variété des réalisations formelles, nous repérons cependant une configuration discursive stable, qui repose sur la jonction syntagmatique de sèmes opposés. Ce «schème paradoxal », qui constitue une "macro-figure " (Aquien \& Molinié, 1999: 243), est susceptible d'actualisations discursives variées, sur des portions du discours plus ou moins grandes (allant du syntagme à la page). Aussi la diversification des formes du paradoxe n'entraine-t-elle pas la dilution de l'esprit paradoxal. Le paradoxe nous apparait au contraire comme une forme matricielle, qui unifie un style à l'échelle du recueil et oriente le regard du moraliste ${ }^{7}$. Il ne s'agit pas de faire du paradoxe l'outil d'un relativisme généralisé, ni d'une dissolution du réel dans le tourbillon des mots, mais de souligner la fonction critique de la figure : le moraliste est un observateur qui, par un jeu de perspective habile, 
pointe les contradictions que dissimulent l'apparente fluidité du théatre social et l'illusoire transparence du discours.

\section{BIBLIOGRAPHIE}

AQUIEN, M. \& MOLINIÉ, G. (1999) : Dictionnaire de rhétorique et de poétique, Paris, Librairie générale française.

AUTHIER-REVUZ, J. (1995) : Ces mots qui ne vont pas de soi. Boucles réflexives et non-coïncidences du dire, Paris, Larousse.

AUTHIER-REVUZ, J., DOURY, M. \& REBOUL-TOURE, S. (éd.) (2003) : Parler des mots : le fait autonymique en discours, Paris, Presses Sorbonne Nouvelle.

Bonнomme, M. (2005) : Pragmatique des figures du discours, Paris, H. Champion.

BURY, E. (1995) : « Une œuvre dans le siècle », in : J. de La Bruyère, Les Caractères, éd. par E. Bury, Paris, Librairie Générale Française, p. 9-42.

COSTENTIN, C. (2003) : Genèse et formes d'une écriture paradoxale dans l'œuvre complète de La Rochefoucauld, thèse, Université de Paris IV.

DANDREY, P. (1997) : L'éloge paradoxal de Gorgias à Molière, Paris, Presses universitaires de France. Guillaume, G. (1929) : Temps et verbe, Paris, H. Champion.

LANDHEER, R. \& SMITH, P. J. (éd.) (1996) : Le Paradoxe en linguistique et en littérature, Genève, Droz. WоŁoWsKA, K. (2008) : Le Paradoxe en langue et en discours, Paris, Éd. L'Harmattan.

\section{NOTES}

1. Pour une étude historique des traditions rhétoriques, littéraires et philosophiques liées au paradoxe, se reporter à P Dandrey (1997).

2. Nous reprenons ici les analyses de J. Authier-Revuz (1995: $805 ; 2003$ : 9) qui décrit la modalité autonymique comme une "rupture liée", ayant pour fonction de souligner «la faille de noncoïncidence énonciative» et d'accomplir dans un même mouvement sa "suture métaénonciative ".

3. Énigme et paradoxe se rencontrent fréquemment, dans la mesure où résoudre une énigme impose souvent de recourir à une interprétation « para-doxale » des termes qui la composent.

4. Nous reprenons ici la distinction proposée par G. Molinié (Aquien \& Molinié, 1999 : 255, 267).

5. L'effet de mention est d'autant plus prégnant que cette expression a déjà fait l'objet des railleries de B. Pascal, dans Les Provinciales (plus précisément dans les lettres I et X).

6. Nous remercions Heike Baldauf-Quilliatre de nous avoir suggéré cet adjectif.

7. Nos recherches sur le paradoxe nous ont convaincu de la pertinence de cette entrée pour l'étude des moralistes de l'âge classique. Notre hypothèse est que le paradoxe constitue ce que l'on pourrait appeler - en référence aux travaux de G. Guillaume (1929: 121-128) - un «schème substylistique » de la prose moraliste. 


\section{RÉSUMÉS}

Cet article étudie le travail d'appropriation d'une figure - le paradoxe - à un régime discursif qui exclut toute forme de grandiloquence : celui de la conversation galante. Dans ce contexte culturel soumis aux codes esthétiques du naturel, la configuration «spectaculaire " du paradoxe pose problème. Nous proposons un parcours des formes que prend «l'acclimatation conversationnelle » du paradoxe dans Les Caractères de La Bruyère et tentons de dégager, derrière la variété des réalisations formelles de la figure, un schème discursif stable, constitutif d'un style.

The present article studies the adaptation of a figure of speech - the paradox - to a discursive regime which prohibits any kind of bombast: the galant conversation of the french salons, in the late 17th Century. In this cultural context, the somewhat "spectacular" configuration of the paradox is problematic. This paper focuses on the different shapes which the "acclimatization" of the paradox takes in La Bruyère's Caractères. It aims to bring out, through the variety of the formal actualizations of the figure, a stable discursive pattern, constituent of a style.

\section{INDEX}

Mots-clés : paradoxe, La Bruyère, stylistique

Keywords : paradox, La Bruyère, stylistics

\section{AUTEUR}

PIERRE-YVES GALLARD

BCL (UMR 7320 CNRS), Université Nice Sophia Antipolis 